\title{
A BOUNDARY VALUE PROBLEM WITH A DISCONTINUOUS COEFFICIENT AND CONTAINING A SPECTRAL PARAMETER IN THE BOUNDARY CONDITION
}

\author{
A. A. DARWISH \\ Mathematics Department, Faculty of Science \\ Qatar University, Doha-Qatar
}

(Received January 5, 1993 and in revised form May 21, 1993)

\begin{abstract}
A singular non-self-adjoint boundary value problem is considered. This problem has a discontinuous coefficient with a spectral parameter in the boundary condition. Some solutions of the eigenvalue equation are given. The discrete spectrum is studied and the resolvent is obtained. Formulation of the adjoint problem is deduced and hence the continuous spectrum of the considered problem is given. Furthermore, the spectrum of the adjoint problem is investigated.
\end{abstract}

KEY WORDS AND PHRASES. Boundary value problems, discontinuous coefficient, spectral parameter, resolvent, adjoint problem.

1991 AMS SUBJECT CLASSIFICATION CODES. 47A, 47E.

\section{INTRODUCTION.}

Consider the boundary value problem corresponding to the modified form of the SturmLiouville equation

$$
-\mathrm{y}^{\prime \prime}+q(x) \text { บ }=\lambda \rho(x) \text { บ }, x \in[0, \infty)
$$

and the boundary condition

$$
\mathrm{Y}^{\prime}(0)-\lambda \sum_{n=1}^{m} \alpha_{n} \mathrm{Y}\left(a_{n}\right)+\lambda \int_{0}^{\infty} G(x) \mathrm{Y}(x) d x=0,
$$

where $\lambda$ is a complex parameter and $\alpha_{n}, a_{n}$ are real constants. We shall assume that the potential function $q(x)$ and the function $G(x)$ are a complex valued, integrable on $[0, \infty)$ and the condition

is satisfied throughout this paper.

$$
\int_{0}^{\infty} x|q(x)| d x<\infty
$$

The function $\rho(x)$ is defined by

$$
\rho(x)= \begin{cases}b^{2}, & 0 \leq x \leq c \\ 1, & c<x<\infty\end{cases}
$$

where $b, c$ are a positive constants and $b \neq 1$.

It is worth noting that the study of boundary value problems containing a spectral parameter in the boundary conditions have many interesting applications, especially in mathematical physics (e.g. [1], pp.146-152). 
Many self-adjoint boundary value problems of this type, for a differential equation of the second order for which $\rho(x) \equiv 1$ and including a spectral parameter in the boundary condition, were discussed in ([3], [4], [6], [7]). In [5] the case of two-point boundary value problems with spectral parameter in the boundary condition was studied. Moreover, a singular non-self-adjoint boundary value problem with a discontinuous coefficient and including a spectral parameter in the boundary condition was investigated in [10].

Our aim in this work is to study a non-self-adjoint boundary value problem, with a discontinuous coefficient, that contains a spectral parameter in the boundary condition.

We give some solutions of equation (1.1) and obtain the Greens function or the resolvent of the problem (1.1)-(1.2). Upon using Lagrange's formula and the resolvent of the problem (1.1)(1.2) we deduce the adjoint problem that associated with that one. Moreover the continuous spectrum of the considered problem is given and then the spectrum of adjoint problem is classified.

\section{SOLUTIONS FOR THE EQUATION (1.1).}

We shall mainly use the basic results that have been obtained in $([8],[9])$. Let us consider first the initial conditions:

where

$$
\left.\begin{array}{rl}
u(c, \kappa)=1, & u^{\prime}(c, \kappa)=0 \\
\checkmark(c, \kappa)=0, & \nabla^{\prime}(c, \kappa)=1
\end{array}\right\}
$$

$$
\kappa=\lambda^{1 / 2}=\sigma+i \tau \quad \text { with } 0 \leq \arg \kappa<\pi .
$$

Upon using the results in [9], it can be shown that the solutions for $x \in[0, c]$, and which satisfy the initial conditions (1.4) can be expressed in the form,

and

$$
u(x, \kappa)=\cos \kappa(x-c) b+\int_{c}^{x} A_{1}(x, t) \cos \kappa(t-c) b d t
$$

$$
\checkmark(x, \kappa)=\frac{\sin \kappa(x-c) b}{\kappa b}+\int_{c}^{x} A_{2}(x, t) \frac{\sin \kappa(t-c) b}{\kappa b} d t,
$$

where the kernels $A_{1}(x, t), A_{2}(x, t)$ satisfies both the differential equations

$$
\frac{\partial^{2} A_{1}}{\partial x^{2}}-\frac{\partial^{2} A_{1}}{\partial t^{2}}=q(x) A_{1}(x, t), \frac{\partial^{2} A_{2}}{\partial x^{2}}-\frac{\partial^{2} A_{2}}{\partial t^{2}}=q(x) A_{2}(x, t)
$$

and the conditions

$$
\begin{gathered}
A_{1}(x, x)=\frac{1}{2} \int_{c}^{x} q(t) d t ;\left.\frac{\partial}{\partial t} A_{1}(x, t)\right|_{t=c} ^{=0} \\
t=c \\
A_{2}(x, x)=\frac{1}{2} \int_{c}^{x} q(t) d t ; A_{2}(x, c)=0 .
\end{gathered}
$$

Moreover, $u(x, \kappa)$ and $\checkmark(x, \kappa)$ are entire functions of $\kappa$ of any fixed $b$ and they have the following asymptotics behavior as $\tau \geq 0,|\kappa| \rightarrow \infty$

$$
\begin{aligned}
& u(x, \kappa)=\cos \kappa(x-c) b+0\left(\frac{1}{\kappa} \exp |\operatorname{Im} \kappa b|(x-c)\right), \\
& \checkmark(x, \kappa)=\frac{\sin \kappa(x-c) b}{\kappa b}+0\left(\frac{1}{\kappa^{2}} \exp |\operatorname{Im} \kappa b|(x-c)\right)
\end{aligned}
$$

uniformly with respect to $x$ on $[0, c]$.

Now, let us denote by

$$
\sigma(x)=\int_{x}^{\infty}|q(t)| d t ; \sigma_{1}(x)=\int_{x}^{\infty} \sigma(t) d t .
$$


For $x \in[c, \infty)$ one can show uning [s] that for any $\kappa$ in the upper half plane the equation $(1.1)$ has a solution of the form

$$
f(x, \kappa)=\epsilon x p(2 \kappa x)+\int_{x}^{\infty} p(x, t) \exp (2 \kappa t) d t .
$$

where $p(x, t)$ satisfies the inequality

$$
|p(x, t)| \leq \frac{1}{2} \sigma\left(\frac{x+t}{2}\right) \exp \left(\sigma_{1}(x)\right)
$$

In addition, if $q(x)$ is differentiable, then $p(x, t)$ is twice differentiable and satisfies both the equation

$$
\frac{\partial^{2} p}{\partial x^{2}}-\frac{\partial^{2} p}{\partial t^{2}}=q(x) p(x, t)
$$

and the condition

$$
\frac{d p(x, x)}{d x}=-\frac{1}{2} q(x)
$$

This solution is an analytic function of $\kappa, \tau>0$ and continuous of $\kappa, \tau \geq 0$. From the continuity of $f(x, \kappa)$ on $[0, c]$ we find

$$
\begin{aligned}
& f(x, \kappa)=f(c, \kappa)\left[\cos \kappa(x-c) b+\int_{c}^{x} A_{1}(x, t) \cos \kappa(t-c) b d t\right] \\
& \quad+f^{\prime}(c, \kappa)\left[\frac{\sin \kappa(x-c) b}{\kappa b}+\int_{c}^{x} A_{2}(x, t) \frac{\sin \kappa(t-c) b}{\kappa b} d t\right]
\end{aligned}
$$

Furthermore, in [8] it was shown that the equation (1.1) has a solution $f_{1}(x, \kappa), x \in[c, \infty)$ such that in a domain $\tau \geq 0,|\kappa|>\delta>0$ the inequality

$$
\left|f_{1}(x, \kappa)\right| \leq M \exp (x \tau)
$$

holds for a sufficiently large $M$. This solution can be written in the form

$$
f_{1}(x, \kappa)=\exp (-i \kappa x)\left(1+0\left(\frac{1}{\kappa}\right)\right)
$$

uniformly with respect to $x>c$ as $|\kappa| \rightarrow \infty$.

Thus, the solutions $f(x, \kappa) ; f_{1}(x, \kappa)$ on $[0, \infty)$ can be written asymptotically as follows:

$$
f(x, \kappa)= \begin{cases}\exp (i \kappa c)\left[\cos \kappa(x-c) b+\frac{i}{b} \sin \kappa(x-c) b\right]\left(1+0\left(\frac{1}{\kappa}\right)\right) & 0 \leq x \leq c \\ \exp (i \kappa x)\left(1+0\left(\frac{1}{\kappa}\right)\right), & c<x<\infty\end{cases}
$$

and

$$
f_{1}(x, \kappa)= \begin{cases}\exp (-i \kappa c)\left[\cos \kappa(x-c) b-\frac{i}{b} \sin \kappa(x-c) b\right]\left(1+0\left(\frac{1}{\kappa}\right)\right), & 0 \leq x \leq c \\ \exp (-i \kappa x)\left(1+0\left(\frac{1}{\kappa}\right)\right), & c<x<\infty .\end{cases}
$$

\section{THE DISCRETE SPECTRUM AND THE RESOLVENT.}

In this respect we study the discrete spectrum and obtain the resolvent of the problem (1.1)(1.2).

The next lemmas 1, 2 follows directly from the work [8].

LEMMA 1. The boundary value problem (1.1)-(1.2) does not have eigenvalues on the 
positive semi-axis $\lambda \geq 0$.

LEMMA 2. The necessary and sufficient conditions that $\lambda \neq 0$ be an eigenvalue of (1.1)(1.2) are that

$$
\lambda=\kappa^{2} ; \tau>0 ; \varphi(\kappa)=f^{\prime}(0, \kappa)-\kappa^{2} \sum_{n=1}^{m} \alpha_{n} f\left(a_{n}, \kappa\right)+\kappa^{2} \int_{0}^{\infty} G(x) f(t, \kappa) d t=0,
$$

It is clear that $\varphi(\kappa)$ is an analytic function in the upper half-plane and this proves the following lemma:

LEMMA 3. The boundary value problem (1.1)-(1.2) has no more than a countable complex set of eigenvalues. The limit points can lie only on the real axis.

Next, the asymptotics formula for investigating the discrete spectrum is obtained.

Taking

$$
\begin{gathered}
\varphi_{0}(\kappa)=\kappa b \exp (i \kappa c)\left[\sin \kappa b c+\frac{l}{b} \cos \kappa b c\right]-\kappa^{2} \sum_{n=1}^{r} \alpha_{n}\left[\cos \kappa b\left(a_{n}-c\right)\right. \\
\left.+\frac{i}{b} \sin \kappa b\left(a_{n}-c\right)\right]-\left(\kappa^{2} \sum_{n=r+1}^{m} \alpha_{n} \exp \left(i \kappa a_{n}\right)\right) .
\end{gathered}
$$

Thus, using (1.9), (2.1) we have

$$
\varphi(\kappa)=\varphi_{0}(\kappa)\left[1+0\left(\frac{1}{\kappa}\right)\right]
$$

Then, for large values of $\kappa$ and $\tau \geq 0$ the functions $\varphi(\kappa)$ and $\varphi_{0}(\kappa)$ are equivalent outside a circle of fixed radius $0<\delta<1$ with a center at the zeros of $\varphi_{0}(\kappa)$,i.e., the circle $D_{\delta}^{n}=\left\{\kappa ;\left|\kappa-\kappa_{n}^{0}\right|<\delta ; 0<\delta<1\right\}$.

Since, $\varphi(\kappa)$ and $\varphi_{0}(\kappa)$ are analytic functions of $\kappa$, by Rouche's theorem they have the same number of finite zeros.

Let $\kappa_{n}, \kappa_{n}^{0}$ be the zeros of $\varphi(\kappa)$ and $\varphi_{0}(\kappa)$ respectively. Thus from (2.3) it is clear that

$$
\kappa_{n} \sim \kappa_{n}^{0} \text { for }|\kappa| \rightarrow \infty \text { with } \tau>0 .
$$

Accordingly, the study of the distribution of eigenvalues of the problem (1.1)-(1.2) is equivalent to the study of the zeros of the function $\varphi_{0}(\kappa)$ in the upper half plane.

Evidently, the function $\varphi_{0}(\kappa)$ does not tend to zero for sufficiently large real values of $\kappa$.

Hence:

THEOREM 1. The set of eigenvalues of the problem (1.1)-(1.2) are bounded in a closed domain in the upper half plane.

THEOREM 2. Let $q(x), G(x)$ satisfy the conditions $\exp (\varepsilon x) q(x) \in \mathcal{L}_{1}(0, \infty)$ and $\exp (\varepsilon x)$ $G(x) \in \mathcal{L}_{1}(0, \infty), \varepsilon>0$.

Then the discrete spectrum of (1.1)-(1.2) consists of a finite number of complex eigenvalues and a possible finite number of real spectral singularities on the positive semi-axis $\lambda \geq 0$.

PROOF. From Lemma 3 it is sufficient to show that the set of complex zeros of $\varphi(\kappa), \tau>0$ has no limit points on $[0, \infty)$. Since for $\lambda=\kappa^{2}, \tau \geq 0$ and $\lambda \in[0, \infty)$ equation (1.1) has a solution of the form (1.5)-(1.7). Thus in view of (1.6) and the assumptions of the theorem we have

$$
|p(x, \kappa)|<M \exp (-\varepsilon / 2[x+t])
$$

where $M$ is a positive constant. Suppose that

$$
\left|A_{1}(x, t)\right|,\left|A_{2}(x, t)\right| \leq M
$$


and the kernels $A_{1}(x, t), A_{2}(x, t)$ have continuous partial derivatives.

Also, the kernel $p(x, t)$ has continuous partial derivatives and

$$
\int_{x}^{\infty}\left[\left|p_{x}^{\prime}(x, t)\right|+\left|p_{t}^{\prime}(x, t)\right|\right] \exp \left({ }^{\varepsilon} / 2 t\right) d t<\infty .
$$

Taking into account (2.3), $(2.4)$ and (2.5) show the integral in (2.1) converges for $\tau>^{\varepsilon} / 2$. By the uniqueness of analytic continuation, the function $f(x, \kappa)$ is not only a solution of (1.1) for $\tau \geq 0$ but also for $\tau>-{ }^{\varepsilon} / 2$. Thus, $\varphi(\kappa)$ is a holomorphic function in a domain $S_{e}=\left\{\kappa: \tau>-{ }^{\varepsilon} / 2\right\}$.

Therefore, the set of complex zeros of $\varphi(\kappa)$ has no limit points on the real axis. Hence, this set is finite in a bounded domain. Moreover, it is possible for $\varphi(\kappa)$ to have a finite number of real zeros as well as a singular spectrum on the positive semi-axis $\lambda \geq 0$ and hence the theorem is proved.

In the sequel, we obtain the resolvent formula of (1.1)-(1.2). If $2 i \kappa f^{\prime}(o, \kappa) \neq 0$, let us define

$$
R_{0}(x, t, \kappa)= \begin{cases}\frac{f_{1}^{\prime}(0, \kappa) f(x, \kappa) f(t, \kappa)}{2 i \kappa f^{\prime}(0, \kappa)}-\frac{1}{2 i \kappa} f(x, \kappa) f_{1}(t, \kappa), & t \leq x \\ \frac{f_{1}^{\prime}(0, \kappa) f(x, \kappa) f(t, \kappa)}{2 i \kappa f^{\prime}(0, \kappa)}-\frac{1}{2 i \kappa} f_{1}(x, \kappa) f(t, \kappa), & t \geq x .\end{cases}
$$

THEOREM 3. If $\lambda=\kappa^{2}$ is not an eigenvalue of (1.1)-(1.2) then the Green's function for

$$
-\mathrm{y}^{\prime \prime}+q(x) \text { บ }-\lambda \rho(x) \text { บ }=\rho f, \quad x \in[0, \infty)
$$

is $R(x, t, \kappa)$. That is, if $f \in \mathcal{L}_{2}(0, \infty, \rho(x))$ then

where

$$
\mathrm{Y}(x, \kappa)=\int_{0}^{\infty} R(x, t, \kappa) \rho(t) f(t) d t,
$$

$$
\left.R(x, t, \kappa)=R_{0}(x, t, \kappa)+\frac{\kappa^{2} f(x, \kappa)}{\varphi(\kappa)}\left[\sum_{n=1}^{m} \alpha_{n} R_{0}\left(a_{n}, t, \kappa\right)+\int_{0}^{\infty} G(x) R_{0}(x, t, \kappa)\right] d x\right] .
$$

PROOF. It is clear from Theorem 1 that all numbers $\lambda=\kappa^{2}, \varphi(\kappa) \neq 0$ and $\tau>0$ are in the resolvent set of the problem (1.1)-(1.2). Since, $\lambda=\kappa^{2}$ is assumed not to be an eigenvalue of (1.1)(1.2), we have the resolvent $R(x, t, \kappa)$ exists. Consequently, there exists a solution of equation (2.7) in $\ell_{2}(0, \infty ; \rho(x))$. Suppose that

$$
\mathrm{y}_{0}(x, \kappa)=\int^{\infty} R_{0}(x, t, \kappa) \rho(t) f(t) d t
$$

is a solution of equation (2.7) that satisfies the condition $\mathrm{Y}^{\prime}(0)=0$. Then, we find $R_{0}(x, t, \kappa)$ is of the form (2.6); therefore, the general solution of the equation (2.7) that belongs to $\ell_{2}(0, \infty ; \rho(x))$ takes the form

$$
\mathrm{y}(x, \kappa)=\mathrm{Y}_{0}(x, \kappa)+C f(x, \kappa),
$$

where $C$ is an arbitrary constant to be found. Since the function บ $(x, \kappa)$ satisfies condition (1.2) we have

$$
C=\frac{\kappa^{2}}{\varphi(\kappa)}\left[\sum_{n=1}^{m} \alpha_{n} \mathcal{Y}_{0}\left(a_{n}, \kappa\right)+\int_{0}^{\infty} G(x) \mathcal{Y}_{0}(x, \kappa) d x\right]
$$

Substituting in (2.9) we deduce that

$$
\mathrm{y}(x, \kappa)=\int_{0}^{\infty} R(x, t, \kappa) \rho(t) f(t) d t
$$


where $R(x, t, \kappa)$ is defined by the formula (2.S).

Furthermore, for $t \leq x$ we have

$$
\begin{aligned}
& R(x, t ; \kappa)= \frac{f(x, \kappa)}{2 \imath \kappa f^{\prime}(0, \kappa)}\left[f_{1}^{\prime}(0, \kappa) f(t, \kappa)-f^{\prime}(0, \kappa) f_{1}(t, \kappa)\right] \\
& \quad+\frac{\kappa f(x, \kappa)}{2 \imath \varphi(\kappa) f^{\prime}(0, \kappa)} \sum_{n=1}^{m} a_{n} f\left(a_{n}, \kappa\right)\left[f_{1}^{\prime}(0, \kappa) f(t, \kappa)-f_{1}(t, \kappa) f^{\prime}(0, \kappa)\right] \\
& \quad+\frac{\kappa f(x, \kappa) f_{1}^{\prime}(0, \kappa) f(t, \kappa)}{2 \imath \varphi(\kappa) f^{\prime}(0, \kappa)} \int_{0}^{\infty} G(x) f(x, \kappa) d x \\
&-\frac{\kappa f(x, \kappa)}{2 i \varphi(\kappa)}\left[f(t, \kappa) \int_{0}^{t} G(x) f_{1}(x, \kappa) d x+f_{1}(t, \kappa) \int_{t}^{\infty} G(x) f(x, \kappa) d x\right]
\end{aligned}
$$

and for $t \geq x$ we have

$$
\begin{aligned}
& R(x, t ; \kappa)= \frac{f(t, \kappa)}{2 i \kappa f^{\prime}(0, \kappa)}\left[f_{1}^{\prime}(0, \kappa) f(x, \kappa)-f^{\prime}(0, \kappa) f_{1}(x, \kappa)\right]+\frac{\kappa f(x, \kappa) f(t, \kappa)}{2 \imath \varphi(\kappa) f^{\prime}(0, \kappa)} \\
& {\left[\sum_{n=1}^{m} \alpha_{n} f\left(a_{n}, \kappa\right) f_{1}^{\prime}(0, \kappa)-\sum_{n=1}^{m} \alpha_{n} f_{1}\left(a_{n}, \kappa\right) f^{\prime}(0, \kappa)\right] } \\
&+ \frac{\kappa f_{1}^{\prime}(0, \kappa) f(x, \kappa) f(t, \kappa)}{2 i \varphi(\kappa) f^{\prime}(0, \kappa)} \int_{0}^{\infty} G(x) f(x, \kappa) d x \\
&-\frac{\kappa f(x, \kappa)}{2 i \varphi(\kappa)}\left[f(t, \kappa) \int_{0}^{t} G(x) f_{1}(x, \kappa) d x+f_{1}(t, \kappa) \int_{t}^{\infty} G(x) f(x, \kappa) d x\right]
\end{aligned}
$$

\section{THE ADJOINT PROBLEM OF (1.1)-(1.2) AND ITS SPECTRUM.}

Now, we consider the resolvent $R(x, t, \kappa)$ of the problem (1.1)-(1.2) to obtain the adjoint problem.

Let us denote by $L_{\lambda}^{*}$ the adjoint operator of the $L_{\lambda}$ operator which is generated by (1.1)(1.2).

We denote by $D\left(L_{\lambda}^{*}\right)$ those functions $\mathscr{Z}$ denoted on $[0, \infty)$ and satisfying:

(i) $\mathscr{Z}$ is in $\mathscr{L}_{2}(0, \infty, \rho(x))$;

(ii) $\mathcal{Z}^{\prime}$ exists and is absolutely continuous on every finite subintervals

$\left[0, a_{1}\right),\left(a_{1}, a_{2}\right), \cdots\left(a_{n}-1, a_{n}\right)$ and $\left(a_{n}, \infty\right)$;

(iii) $\overline{\mathscr{L}}^{\prime}\left(a_{n}+0\right)-\overline{\mathscr{G}}^{\prime}\left(a_{n}-0\right)=\alpha_{n} \lambda \overline{\mathscr{W}}(0)$;

(iv) $\overline{\mathscr{Z}}^{\prime}(0)=0$;

(v) $\mathscr{Z}$ is twice differentiable as $x \neq a_{n}, n=\overline{1, m}$ and $-\overline{\mathscr{Z}}^{\prime \prime}+\bar{q}(x) \mathscr{Z} \in \mathcal{L}_{2}(0, \infty ; \rho(x))$.

THEOREM 4. Assume that $\mathscr{Z} \in D\left(L_{\lambda}^{*}\right)$ satisfies the conditions (i)-(v). Then the adjoint problem of (1.1)-(1.2) can be written in the form

$$
\begin{aligned}
& -\overline{\mathscr{L}}^{\prime \prime}+\bar{q}(x) \mathscr{Z}-\lambda\left[\bar{G}(x)-\sum_{n=1}^{m} \alpha_{n} \delta\left(x-a_{n}\right)\right] \mathscr{Z}(0)=\lambda \rho \mathscr{Z}, \\
& \overline{\mathscr{L}}^{\prime}(0)=0 .
\end{aligned}
$$

PROOF. If $\mathcal{Y} \in D\left(L_{\lambda}\right)$ and $\mathscr{Z} \in D\left(L_{\lambda}^{*}\right)$ then we have $\left(L_{\lambda}\right.$ บ, $\left.\mathscr{Z}\right)=\int_{0}^{\infty}\left(-\mathrm{Y}^{\prime \prime}+q(x)\right.$ บ $)$

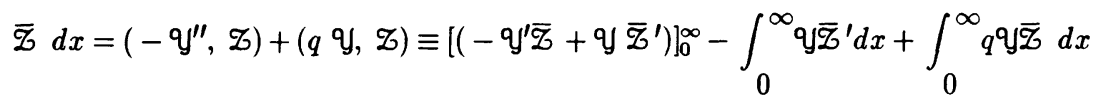




$$
\begin{aligned}
& =\mathcal{Y}^{\prime}(0) \overline{\mathscr{Z}}(0)-\mathcal{Y}(0) \overline{\mathscr{L}}^{\prime}(0)+\int_{0}^{x}\left(-\overline{\mathscr{L}}^{\prime \prime}+q \overline{\mathscr{Z}}\right) \text { ข } d x \\
& -\int_{0}^{\infty}\left(-\overline{\mathscr{Z}}^{\prime \prime}+q \overline{\mathscr{Z}}\right) \text { บ } d x-\text { บ }(0) \overline{\mathscr{Z}}^{\prime}(0)+\kappa^{2} \overline{\mathscr{Z}}(0)\left[\sum_{n=1}^{m} \alpha_{n} \text { บ }\left(a_{n}\right)\right. \\
& \left.\left.-\int_{0}^{\infty} G(x) \text { บ }(x) d x\right]=\int_{0}^{\infty}\left(-\overline{\mathscr{Z}}^{\prime \prime}+q \overline{\mathscr{Z}}\right)-\kappa^{2} \overline{\mathscr{Z}}(0) G(x)\right) \text { ข } d x \\
& +\kappa^{2} \overline{\mathscr{Z}}(0) \sum_{n=1}^{m} \alpha_{n} \int_{0}^{\infty} \delta\left(x-a_{n}\right) \text { ข } d x-\text { ข }(0) \overline{\mathscr{J}}^{\prime}(0) \\
& =\int_{0}^{\infty}\left(-\overline{\mathscr{Z}}^{\prime \prime}+q \overline{\mathscr{Z}}-\kappa^{2} \overline{\mathscr{Z}}(0)\left[G(x)-\sum_{n=1}^{m} \alpha_{n} \delta\left(x-a_{n}\right)\right] \text { ข } d x-\text { ข }(0) \overline{\mathscr{Z}}^{\prime}(0)\right. \text {. }
\end{aligned}
$$

Next, we show that $\overline{\mathscr{L}}^{\prime}(0)=0$. Since

$$
\mathscr{Z}(x)={ }_{0} \int^{\infty} \overline{R(t, x, \bar{\kappa})} \rho(t) f(t) d t, f(t) \in \mathcal{L}_{2}(0, \infty, \rho(x))
$$

we have

$$
\overline{\mathcal{Z}}^{\prime}(0)=\int^{\infty} R_{t}^{\prime}(0, x, \bar{\kappa}) \rho(t) f(t) d t .
$$

In fact, $R(x, t, \kappa)$ as $t \leq x$ has the form (2.10). By differentiation with respect to $t$ we obtain

$$
\begin{aligned}
& R_{t}^{\prime}(0, x, \bar{\kappa})=\frac{f(x, \bar{\kappa})}{2 i \kappa f^{\prime}(0, \kappa)}\left[f_{1}^{\prime}(0, \bar{\kappa}) f^{\prime}(0, \bar{\kappa})-f^{\prime}(0, \bar{\kappa}) f_{1}^{\prime}(0, \bar{\kappa})\right] \\
& \quad+\frac{\kappa f(x, \bar{\kappa})}{2 i \varphi(\bar{\kappa}) f^{\prime}(0, \bar{\kappa})} \sum_{n=1}^{m} \alpha_{n} f\left(a_{n}, \bar{\kappa}\right)\left[f_{1}^{\prime}(0, \bar{\kappa}) f^{\prime}(0, \bar{\kappa})-f_{1}^{\prime}(0, \bar{\kappa}) f^{\prime}(0, \bar{\kappa})\right] \\
& \quad+\frac{\kappa f(x, \bar{\kappa}) f_{1}^{\prime}(0, \bar{\kappa})}{2 i \varphi(\bar{\kappa})} \int_{0}^{\infty} G(x) f(x, \bar{\kappa}) d x \\
& \quad-\frac{\kappa f(x, \bar{\kappa}) f_{1}^{\prime}(0, \bar{\kappa})}{2 i \varphi(\bar{k})} \int_{0}^{\infty} G(x) f(x, \bar{\kappa}) d x=0 \text { thus } R_{t}^{\prime}(0, x, \bar{k})=0
\end{aligned}
$$

Similarly, for $t \geq x$ we can show that $R_{t}^{\prime}(0, x, \bar{\kappa})=0$ by using (2.11).

$$
\begin{aligned}
\left(L_{\lambda} \text { บ }, \mathscr{Z}\right) & =\int_{0}^{\infty}\left(-\overline{\mathscr{Z}}^{\prime \prime}+q \overline{\mathscr{Z}}-\kappa^{2} \overline{\mathscr{Z}}(0)\left[G(x)-\sum_{n=1}^{m} \alpha_{n} \delta\left(x-a_{n}\right)\right) \text { บ } d x\right. \\
& =\left(\mathcal{Y},-\overline{\mathscr{Z}}^{\prime \prime}+\bar{q} \mathscr{Z}-\kappa^{2} \mathscr{Z}(0)\left[\bar{G}(x)-\sum_{n=1}^{m} \alpha_{n} \delta\left(x-a_{n}\right)\right)=\left(\text { บ }, L_{\lambda}^{*} \mathscr{Z}\right) .\right.
\end{aligned}
$$

Hence, the adjoint problem takes the form

$$
\begin{aligned}
& -\overline{\mathscr{L}}^{\prime \prime}+\bar{q}(x) \mathscr{Z}-\lambda\left[\bar{G}(x)-\sum_{n=1}^{m} \alpha_{n} \delta\left(x-a_{n}\right)\right] \mathscr{Z}(0)=\lambda \rho Z, \\
& \overline{\mathscr{L}}^{\prime}(0)=0,
\end{aligned}
$$

where $\mathscr{Z}$ satisfies the properties (i)-(v).

THEOREM 5. The positive semi-axis $\lambda \geq 0$ constitutes a continuous spectrum of (1.1)-(1.2) unless both $\varphi(\kappa)$ and $\varphi(-\kappa)$ vanish simultaneously. This theorem can be proved by using the adjoint problem (3.1)-(3.2) and the work [8]. 
Upon using Lemma 2 and Theorem 5 the next theorem follows directly from [2]:

THEOREM 6. The spectrum of (3.1)-(3.2) consists of:

(i) eigenvalues $\bar{\lambda}$ whenever $\varphi(\kappa)=0, \tau>0$ and $\lambda=\kappa^{2}$ on the positive semi-axis $\lambda \geq 0$,

(ii) continuous spectrum on the positive semi-axis $\lambda \geq 0$ unless both $\varphi(\kappa)$ and $\varphi(-\kappa)$ vanish simultaneously.

We claim that all numbers $\bar{\lambda}$ whenever $\varphi(\kappa) \neq 0$ and $\lambda=\kappa^{2}$ from the upper half plane belong to the resolvent set of the problem (3.1)-(3.2) and its resolvent is $\bar{R}(t, x, \bar{\kappa})$.

ACKNOWLEDGEMENT. The author is extremely thankful to Prof. M.G. Gasymov, Professor of Mathematics in Azerbaidjan University for his comments on this paper.

\section{REFERENCES}

1. TIKHNOW, A.H. and SAMARSKU, A.A., Equations of Mathematical Physics, Moscow, 1953.

2. KRALL, A.M., The adjoint of a differential operator with integral boundary condition, Proc. Amer. Math. Soc. 16 (1965), 738-742.

3. WALTER, J., Regular eigenvalue problem with eigenvalue parameter in the boundary conditions, Math. Z. 133 (1973), 301-312.

4. SCHNEIDER, A., A note on eigenvalue problems with eigenvalue parameter in the boundary conditions, Math. Z., 136 (1974), 163-167.

5. FULTON, C.T., Two point boundary value problems with eigenvalue parameter contained in the boundary conditions, Proc. Roy. Soc. Edin 77A (1977), 293-308.

6. HINTON, D.B., An expansion theorem for an eigenvalue problem with eigenvalue parameter in the boundary condition, Quart. J. Math. Oxford 30 N2 (1979), 33-42.

7. SHKALUKOV, A.A., Boundary value problem for ordinary differential equation with parameter in the boundary condition; Trudy cemunara Um. U.G. Petrovskovo 9, Moscow (Russ), 1983.

8. NAIMARK, M.A., Linear differential operators, Frederick Ungar Publishing Co., Inc., London, 1968.

9. MARCHENKO, V.A., Sturm Liouville operators and applications, Birkhauser Verlag Basel, 1986.

10. DARWISH, A.A., On a non-self adjoint singular boundary value problem, Kyungpook Math. J. Taegu, 33 (1) (1993), 1-11. 


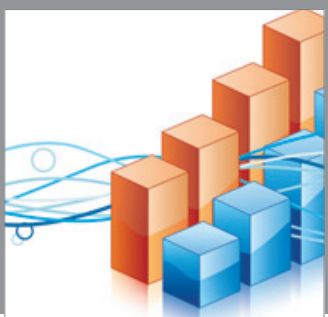

Advances in

Operations Research

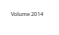

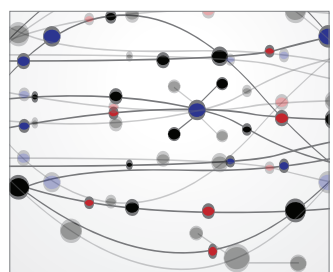

\section{The Scientific} World Journal
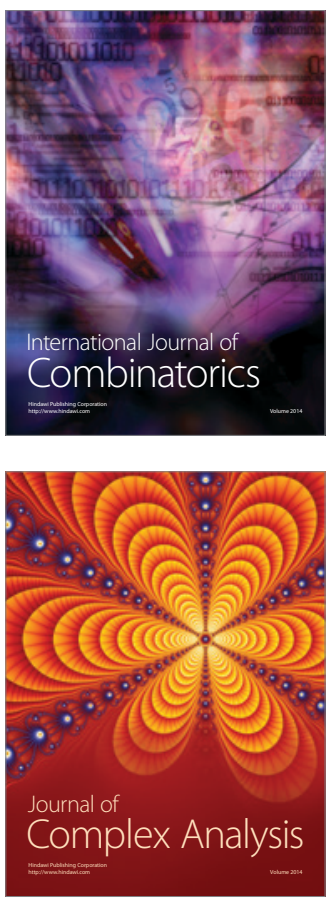

International Journal of

Mathematics and

Mathematical

Sciences
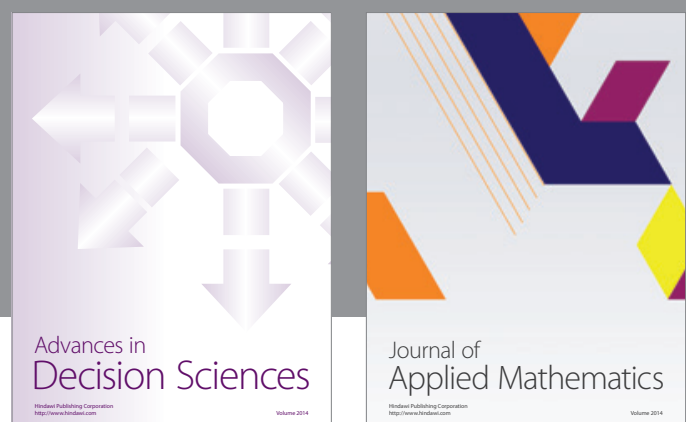

Journal of

Applied Mathematics
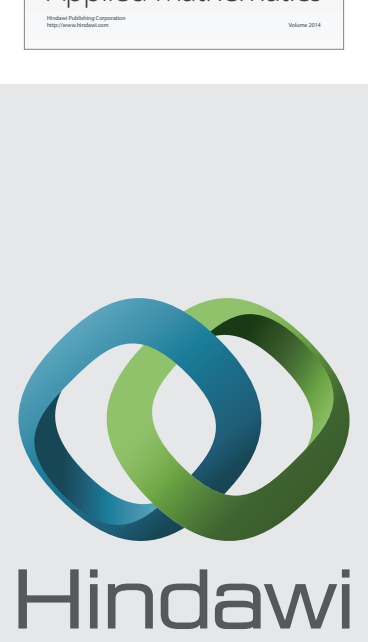

Submit your manuscripts at http://www.hindawi.com
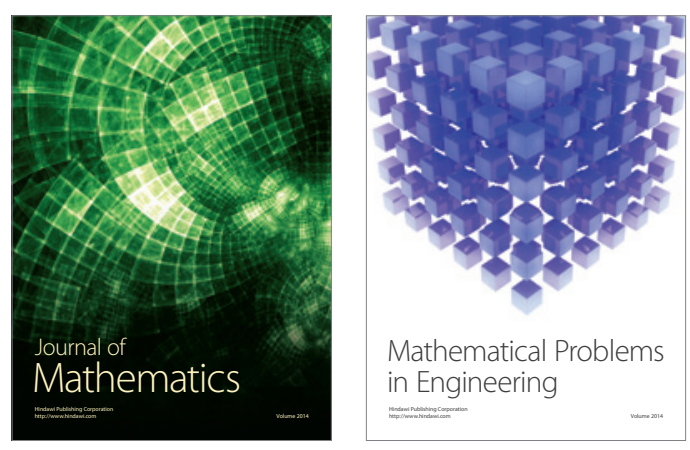

Mathematical Problems in Engineering
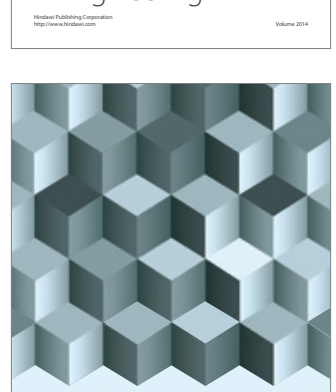

Journal of

Function Spaces
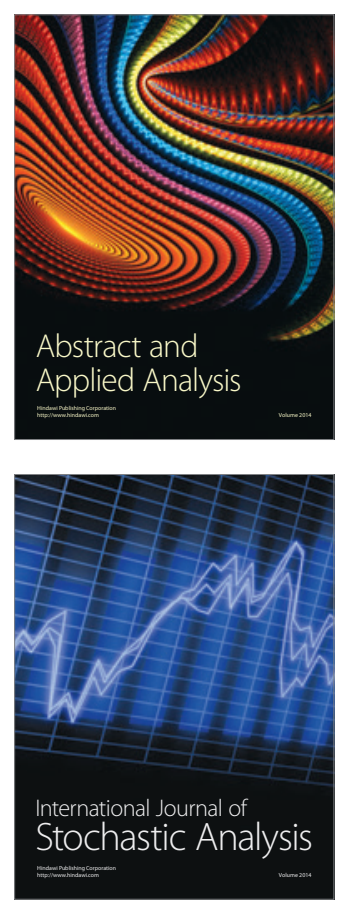

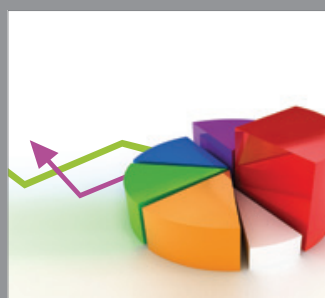

ournal of

Probability and Statistics

Promensencen
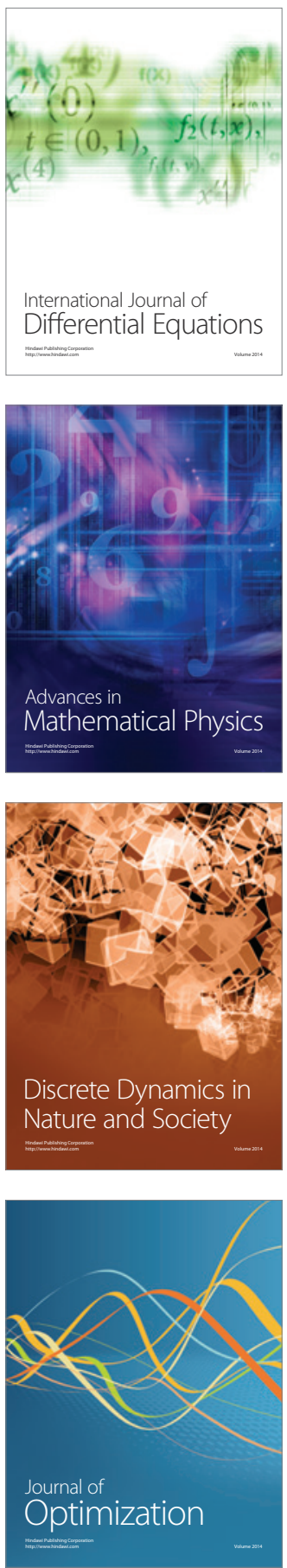